\title{
CONFIRMATION OF CIRCUMSTELLAR PHOSPHINE
}

\author{
M. Agúndez ${ }^{1}$, J. Cernicharo ${ }^{1}$, L. Decin ${ }^{2,3}$, P. Encrenaz ${ }^{4}$, and D. Teyssier ${ }^{5}$ \\ ${ }^{1}$ Instituto de Ciencia de Materiales de Madrid, CSIC, C/ Sor Juana Inés de la Cruz 3, E-28049 Cantoblanco, Spain \\ 2 Sterrenkundig Instituut Anton Pannekoek, University of Amsterdam, Science Park 904, NL-1098 Amsterdam, The Netherlands \\ ${ }^{3}$ Instituut voor Sterrenkunde, Katholieke Universiteit Leuven, Celestijnenlaan 200D, B-3001 Leuven, Belgium \\ ${ }^{4}$ LERMA, Observatoire de Paris, 61 Av. de l'Observatoire, F-75014 Paris, France \\ ${ }^{5}$ European Space Astronomy Centre, Urb. Villafranca del Castillo, P.O. Box 50727, E-28080 Madrid, Spain \\ Received 2014 June 16; accepted 2014 July 3; published 2014 July 16
}

\begin{abstract}
Phosphine $\left(\mathrm{PH}_{3}\right)$ was tentatively identified a few years ago in the carbon star envelopes IRC +10216 and CRL 2688 from observations of an emission line at $266.9 \mathrm{GHz}$ attributable to the $J=1-0$ rotational transition. We report the detection of the $J=2-1$ rotational transition of $\mathrm{PH}_{3}$ in IRC +10216 using the HIFI instrument on board Herschel, which definitively confirms the identification of $\mathrm{PH}_{3}$. Radiative transfer calculations indicate that infrared pumping in excited vibrational states plays an important role in the excitation of $\mathrm{PH}_{3}$ in the envelope of IRC +10216 , and that the observed lines are consistent with phosphine being formed anywhere between the star and $100 R_{*}$ from the star, with an abundance of $10^{-8}$ relative to $\mathrm{H}_{2}$. The detection of $\mathrm{PH}_{3}$ challenges chemical models, none of which offer a satisfactory formation scenario. Although $\mathrm{PH}_{3}$ holds just $2 \%$ of the total available phosphorus in IRC +10216 , it is, together with HCP, one of the major gas phase carriers of phosphorus in the inner circumstellar layers, suggesting that it could also be an important phosphorus species in other astronomical environments. This is the first unambiguous detection of $\mathrm{PH}_{3}$ outside the solar system, and is a further step toward a better understanding of the chemistry of phosphorus in space.
\end{abstract}

Key words: astrochemistry - circumstellar matter - line: identification - molecular processes - stars: AGB and post-AGB - stars: individual (IRC +10216)

Online-only material: color figure

\section{INTRODUCTION}

Phosphorus is a biogenic element, so studying the various forms in which it is present in different astronomical environments is a matter of great interest, although still poorly understood (Maciá 2005). Only five P-containing molecules (PN, CP, $\mathrm{HCP}, \mathrm{PO}$, and $\mathrm{C}_{2} \mathrm{P}$ ) have been observed in the gas phase of interstellar and circumstellar media (Turner \& Bally 1987; Ziurys 1987; Guélin et al. 1990; Agúndez et al. 2007; Tenenbaum et al. 2007; Halfen et al. 2008). These five molecules are all observed in circumstellar envelopes around evolved stars. However, in interstellar clouds only PN is observed and it has a relatively low abundance, implying that most of the phosphorus must be elsewhere, probably depleted in dust grains (Turner et al. 1990).

Phosphine $\left(\mathrm{PH}_{3}\right)$, the phosphorus cousin of ammonia, is a relatively stable molecule that could be holding an important fraction of the phosphorus in various astronomical environments. Since more than $30 \mathrm{yr}$ ago, $\mathrm{PH}_{3}$ has been known to be present in the atmospheres of the giant gaseous planets Jupiter and Saturn, where it is the major phosphorus carrier (Bregman et al. 1975; Larson et al. 1977; Weisstein \& Serabyn 1996). Upper limits obtained in Neptune and Uranus imply that the gas phase abundance of phosphorus is probably subsolar in the atmospheres of these icy giants (Moreno et al. 2009). Phosphine ice is also a plausible major phosphorus constituent of comets, although recent searches in the gaseous coma of a few comets were unsuccessful, providing upper limits that were not significant enough to conclude whether or not $\mathrm{PH}_{3}$ holds most of phosphorus in these solar system bodies (Crovisier et al. 2004; Agúndez et al. 2014).

Outside the solar system, no evidence has been found of the presence of $\mathrm{PH}_{3}$ in interstellar clouds, either in the gas phase or as ice in dust grains. There is, however, some evidence of $\mathrm{PH}_{3}$ being present in the outflows of evolved stars. A few years ago this molecule was tentatively identified in the carbon star envelope IRC +10216 . The identification was based on the $J=1-0$ rotational transition, lying at $266.9 \mathrm{GHz}$, which, however, appeared contaminated by a narrow line assigned to SiS in its $v=4$ vibrational state (Agúndez et al. 2008). An emission feature attributable to the $J=1-0$ line of $\mathrm{PH}_{3}$ was also observed independently by Tenenbaum \& Ziurys (2008) in the spectra of IRC +10216 and the carbon-rich envelope CRL 2688. The observation of just one line, affected by blending in the case of IRC +10216 , led them to consider the detection of $\mathrm{PH}_{3}$ as tentative. Here, we report the detection of the $J=2-1$ rotational transition of $\mathrm{PH}_{3}$ in IRC +10216 using the HIFI instrument on board Herschel, ${ }^{6}$ which definitively confirms the identification of phosphine in this source. This is the first time that $\mathrm{PH}_{3}$ is unambiguously observed outside the solar system.

\section{OBSERVATIONS: IDENTIFICATION OF $\mathrm{PH}_{3}$}

The HIFI observations of IRC +10216 were obtained in 2010 May and from October to December, in the context of two GT1 programs dedicated to performing a line survey in all HIFI bands and searching for light hydrides at selected frequencies. Data were taken in double beam-switching mode with a spectral resolution of $1.1 \mathrm{MHz}$ and a channel spacing of $0.5 \mathrm{MHz}$, and processed using the standard Herschel pipeline up to level 2, which provides fully calibrated spectra in the antenna temperature scale. The intensity scale was later transformed to the main beam brightness temperature $T_{\mathrm{MB}}$. The local oscillator was shifted in frequency to identify any emission arising from

\footnotetext{
6 Herschel is an ESA space observatory with science instruments provided by European-led Principal Investigator consortia and with important participation from NASA.
} 

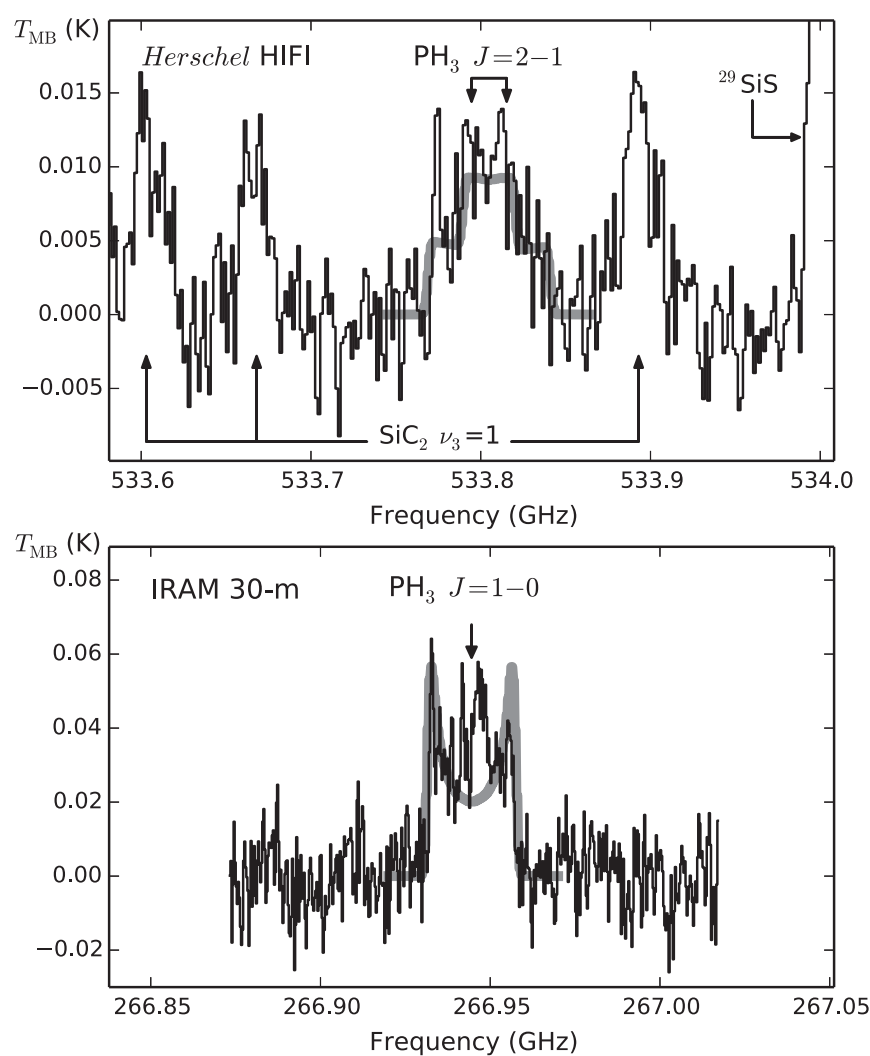

Figure 1. $\mathrm{PH}_{3}$ lines observed in IRC +10216 (black histogram). The lower panel shows the $J=1-0$ transition at $266.9 \mathrm{GHz}$ observed with the IRAM $30 \mathrm{~m}$ telescope at a spectral resolution of $0.32 \mathrm{MHz}$. An overlapping narrow line arising from SiS $v=4$ has been subtracted (see Agúndez et al. 2008). The upper panel shows the $J=2-1$ transition (consisting of $K=0$ and $K=1$ components) at $533.8 \mathrm{GHz}$ observed with Herschel HIFI. The spectrum, smoothed to a spectral resolution of $1.5 \mathrm{MHz}$, also shows three lines of $\mathrm{SiC}_{2}$ $v_{3}=1$ and one line of ${ }^{29} \mathrm{SiS}$. Thick gray lines are the line profiles calculated with the LVG model (see Section 3). The central peak observed in the $J=1-0$ line, not accounted for by the model, is probably due to a non-optimal subtraction of the SiS $v=4$ overlapping line.

the image band. Spectra were smoothed to a spectral resolution of $1.5 \mathrm{MHz}$. For details about the data reduction, we refer to Cernicharo et al. (2010).

Phosphine was searched for in IRC +10216 with HIFI through its $J=2-1$ and $J=3-2$ rotational transitions. This molecule is an oblate symmetric rotor and thus its rotational levels are defined by the quantum numbers $J$ and $K$, and radiative transitions are only allowed within levels of the same $K$ ladder. The $K$ ladders are grouped into two distinct forms: ortho and para, between which radiative and collisional transitions are severely forbidden. The rotational spectrum of $\mathrm{PH}_{3}$ has been extensively studied in the laboratory and line frequencies are accurately known (Cazzoli \& Puzzarini 2006; Müller 2013; Sousa-Silva et al. 2013). Its electric dipole moment has been measured as 0.574 D (Davies et al. 1971).

The $J=2-1$ rotational transition of $\mathrm{PH}_{3}$ consists of two $K$ components: $2_{0}-1_{0}$ at $533.7946 \mathrm{GHz}$ and $2_{1}-1_{1}$ at $533.8153 \mathrm{GHz}$. The HIFI spectrum of IRC +10216 at these frequencies is shown in the upper panel of Figure 1. The $T_{\mathrm{MB}} \mathrm{rms}$ noise level is $0.0024 \mathrm{~K}$ per $1.5 \mathrm{MHz}$ channel. There is an emission feature with $T_{\mathrm{MB}} \sim 0.01 \mathrm{~K}$ centered at $533.8 \mathrm{GHz}$, which can be clearly identified with a composite of the two $K$ components of the $J=2-1$ transition of $\mathrm{PH}_{3}$. We have verified that there is no other obvious assignment for this emission feature by looking at the spectral catalog of
MADEX (Cernicharo 2012). Each of the two $K$ components has a line width consistent with an expansion velocity $v_{\text {exp }}$ of $14.5 \mathrm{~km} \mathrm{~s}^{-1}$, as do most of the lines arising from the outer envelope in IRC +10216 (Cernicharo et al. 2000), including the line previously observed with the IRAM $30 \mathrm{~m}$ telescope and assigned to the $J=1-0$ transition of $\mathrm{PH}_{3}$ (see the lower panel of Figure 1 and Agúndez et al. 2008). The spectrum around $533.8 \mathrm{GHz}$ also shows three narrow lines, with $v_{\exp } \sim$ $8-10 \mathrm{~km} \mathrm{~s}^{-1}$, arising from $\mathrm{SiC}_{2}$ in its $v_{3}=1$ vibrational state and one from ${ }^{29} \mathrm{SiS}$. Lines from these species have been observed already in IRC +10216 at millimeter wavelengths (Cernicharo et al. 2000; Agúndez et al. 2012) and are also detected across the HIFI spectral scan.

The $J=3-2$ transition of $\mathrm{PH}_{3}$ lying at $800.5 \mathrm{GHz}$ is not detected because the sensitivity of the HIFI spectrum at these frequencies is not good enough. The $T_{\mathrm{MB}} \mathrm{rms}$ noise level is $20 \mathrm{mK}$ per $1.5 \mathrm{MHz}$ channel and this $\mathrm{PH}_{3}$ line is expected to have a $T_{\mathrm{MB}}$ of some $\mathrm{mK}$. Moreover, even if the sensitivity were much better, the detection of this line would be hampered by the fact that the $K=0$ and $K=1$ components, at $800.4562 \mathrm{GHz}$ and $800.4871 \mathrm{GHz}$, would appear severely blended with two strong lines from $\mathrm{SiC}_{2}\left(34_{10,25}-33_{10,24}\right.$ and $34_{10,24}-33_{10,23}$ at $800.4842 \mathrm{GHz}$ and $800.4907 \mathrm{GHz}$; Müller et al. 2012). Intense lines from warm $\mathrm{SiC}_{2}$ are numerous across the whole HIFI spectrum (Cernicharo et al. 2010). The $K=2$ component of $\mathrm{PH}_{3}$ at $800.5799 \mathrm{GHz}$ does not overlap with these $\mathrm{SiC}_{2}$ lines (the line widths in IRC +10216's spectra at these frequencies are $\sim 80 \mathrm{MHz}$ ) and thus would be observable if the sensitivity were good enough.

The detection of the $J=2-1$ transition of $\mathrm{PH}_{3}$ with HIFI, together with the previous detection of the $J=1-0$ line (Agúndez et al. 2008; Tenenbaum \& Ziurys 2008), definitively confirms the identification of $\mathrm{PH}_{3}$ in IRC +10216 . The nondetection of the $J=3-2$ line in our HIFI data is consistent with the line intensities of the $J=1-0$ and $J=2-1$ lines, and thus with the identification of $\mathrm{PH}_{3}$.

\section{DISCUSSION}

To extract information about the abundance, spatial distribution, and excitation of $\mathrm{PH}_{3}$ in the envelope of IRC +10216 , the observed lines must be compared with the results of excitation and radiative transfer calculations. Here, the calculations are based on a multi-shell large velocity gradient (LVG) formalism and the model of the envelope adopted is that of Agúndez et al. (2012). Basically, it consists of an asymptotic giant branch (AGB) star surrounded by a spherically expanding envelope of gas and dust. The adopted distance and mass-loss rate are $130 \mathrm{pc}$ and $2 \times 10^{-5} M_{\odot} \mathrm{yr}^{-1}$, and dust is assumed to condense at a radius of $5 R_{*}$. The stellar properties, radial profiles, and dust parameters are described in Agúndez et al. (2012). In the innermost layers $\left(<5 R_{*}\right.$ ), we have adopted the downward revision of the particle density derived by Cernicharo et al. (2013) from ALMA observations of HNC in various excited vibrational states.

Energy levels and transition frequencies of $\mathrm{PH}_{3}$ in its ground vibrational state were computed from the rotational constants reported by Cazzoli \& Puzzarini (2006). Line strengths for pure rotational transitions were computed from the dipole moment measured by Davies et al. (1971). Both ortho and para forms of $\mathrm{PH}_{3}$ were considered with the statistical ortho-to-para ratio of 1. Since rate coefficients for collisional excitation of $\mathrm{PH}_{3}$ with $\mathrm{H}_{2}$ or $\mathrm{He}$ are not known, we adopted those computed for $\mathrm{NH}_{3}$ (Danby et al. 1988; Machin \& Roueff 2005), properly 
corrected to a case without inversion doubling. Scaling of the rate coefficients due to the mass change from $\mathrm{NH}_{3}$ to $\mathrm{PH}_{3}$ is very small $(<5 \%)$ and was thus not considered. No extrapolation was performed for the temperature, and thus the rate coefficients at $300 \mathrm{~K}$, the highest temperature available in the quantum calculations of $\mathrm{NH}_{3}$, were adopted at higher temperatures. The use of these rate coefficients is probably a major source of the uncertainty introduced in the excitation calculations. We included rotational levels up to $J_{K}=7_{6}$ for the ortho species and $J_{K}=5_{5}$ for para $\mathrm{PH}_{3}$, the highest levels involved in quantum calculations of the collisional rate coefficients for $\mathrm{NH}_{3}$. Infrared pumping in excited vibrational states was taken into account by including the first excited states of the four vibrational modes $v_{1}, v_{2}, v_{3}$, and $v_{4}$, lying at 2321, 992, 2327, and $1118 \mathrm{~cm}^{-1}$, respectively, over the ground state. Spectroscopic constants and band intensities were taken from laboratory measurements (Baldacci et al. 1980; Tarrago et al. 1984; Fusina \& Di Lonardo 2000; Brown et al. 2002; Yurchenko et al. 2006; Sousa-Silva et al. 2013). The fundamental bands of these modes lie at 4.3, 9 , and $10 \mu \mathrm{m}$, wavelengths at which the flux in the envelope of IRC +10216 is large (Cernicharo et al. 1999). Collisional rates for rovibrational transitions were assumed to be negligible compared with radiative rates.

The spatial distribution of phosphine in the envelope of IRC +10216 is uncertain because the observed line profiles provide limited information. In the case of the $J=1-0$ line, subtraction of the blended line $\operatorname{SiS} v=4$ introduces an important uncertainty in the real shape of the $\mathrm{PH}_{3}$ contribution. The profile of the $J=2-1$ line is complicated by the overlap of the $K=0$ and $K=1$ components and the limited signalto-noise ratio. In any case, the $\mathrm{PH}_{3}$ lines observed show a width consistent with an expansion velocity of $14.5 \mathrm{~km} \mathrm{~s}^{-1}$, which indicates that an important part of the $\mathrm{PH}_{3}$ emission comes from regions where the gas has already reached the terminal expansion velocity of the envelope. Whether phosphine is formed close to the star or farther out in the envelope is unknown, although there are reasons to suspect that it is not formed too close to the star. On one hand, the abundance of $\mathrm{PH}_{3}$ predicted at thermochemical equilibrium in the hot and dense surroundings of the star is negligible, $<10^{-12}$ relative to $\mathrm{H}_{2}$ (Agúndez et al. 2007). On the other, infrared observations rule out the presence of an important amount of the related molecule $\mathrm{NH}_{3}$ inside a radius of $\sim 20 R_{*}$ (Keady \& Ridgway 1993; Monnier et al. 2000). Following Hasegawa et al. (2006) and Agúndez et al. (2008), here we assume that $\mathrm{PH}_{3}$ is formed at an inner radius of $20 R_{*}$. The outer boundary is calculated with a photochemical model in which $\mathrm{PH}_{3}$ is photodissociated by the external UV field using the rate guessed by MacKay \& Charnley (2001). We find that to reproduce the observed lines (see Figure 1), we need a $\mathrm{PH}_{3}$ abundance of $10^{-8}$ relative to $\mathrm{H}_{2}$, which is slightly higher than the value previously derived by Agúndez et al. (2008) from the $J=1-0$ line. The adopted abundance profile is shown in the upper panel of Figure 2.

Various conclusions can be drawn from a careful inspection of the excitation and radiative transfer calculations. The first is that infrared pumping in excited vibrational states plays an important role in the excitation of $\mathrm{PH}_{3}$ in the envelope. It increases the excitation temperature of rotational transitions within the ground vibrational state, and therefore their line intensities, especially the $J=2-1$ and higher $J$ transitions, and extends the emitting region of the lines to larger radii. This can be appreciated in the lower panel of Figure 2, where we show the contribution to the velocity-integrated line intensity of rays arising at each

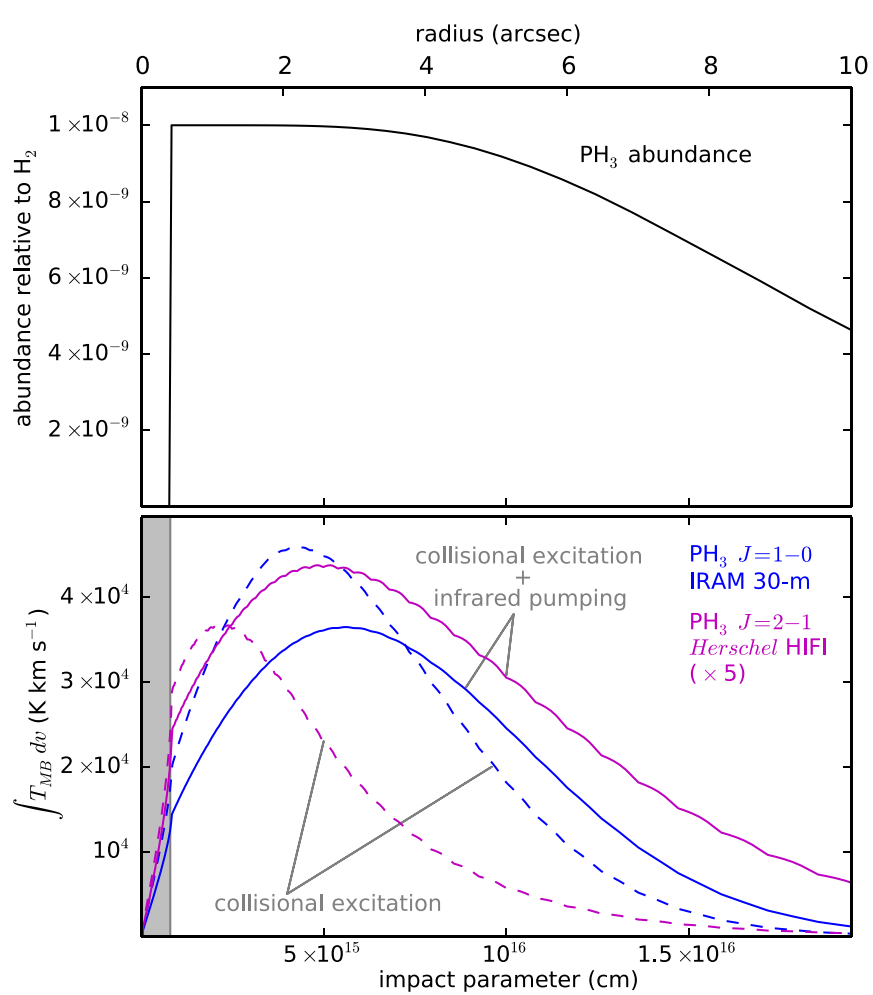

Figure 2. Upper panel: fractional abundance radial profile adopted for $\mathrm{PH}_{3}$. Phosphine is assumed to be present from $20 R_{*}$ with an abundance of $10^{-8}$ relative to $\mathrm{H}_{2}$ and to decrease in the outer layers due to photodissociation by interstellar UV photons. Lower panel: velocity-integrated intensity computed as a function of the impact parameter for the $J=1-0$ and $J=2-1$ lines of $\mathrm{PH}_{3}$. The integration over the impact parameter (if expressed as angular distance in radians) of the magnitude plotted in the ordinate axis yields a calculated value of $\int T_{\mathrm{MB}} d v$. The shaded area corresponds to the region inside $20 R_{*}$. The two panels share a common abscissa axis.

(A color version of this figure is available in the online journal.)

impact parameter. It can be seen that the bulk of the emission in the $J=1-0$ and $J=2-1$ lines comes from regions with impact parameters from the star between $1^{\prime \prime}$ and $6^{\prime \prime}$. That is, the emission size of the $J=1-0$ line is of the order of the main beam of the IRAM $30 \mathrm{~m}$ telescope $\left(9^{\prime \prime} .2\right.$ at $\left.266.9 \mathrm{GHz}\right)$, while the $J=2-1$ line is highly diluted in the main beam of HIFI $\left(40^{\prime \prime}\right.$ at $\left.533.8 \mathrm{GHz}\right)$. We can also appreciate that the observed lines of $\mathrm{PH}_{3}$ do not trace the regions inside $20 R_{*}$. Even if we extended phosphine down to the stellar photosphere, the contribution of impact parameters closer than $20 R_{*}$ to the velocity-integrated line intensities would be less than $5 \%$. In fact, our LVG calculations indicate that the observed lines are compatible with $\mathrm{PH}_{3}$ being formed anywhere between the stellar surface and $\sim 100 R_{*}$.

Assuming that the elemental abundance of phosphorus in IRC +10216 is solar $\left(\mathrm{P} / \mathrm{H}=2.6 \times 10^{-7}\right.$; Asplund et al. 2009), it turns out that HCP holds $5 \%$ of the phosphorus (Agúndez et al. 2007, 2012) while $\mathrm{PH}_{3}$ holds $2 \%$. It is likely that the rest of the $\mathrm{P}$ is in some condensed form as a part of dust grains. In envelopes around other evolved stars, gas phase molecules seem to hold a greater fraction of phosphorus than in IRC +10216 . Around half of the $\mathrm{P}$ is in the form of $\mathrm{HCP}$ and $\mathrm{PH}_{3}$ in the carbon-rich envelope CRL 2688 (Milam et al. 2008; Tenenbaum \& Ziurys 2008), while in oxygen-rich objects PO and PN contain a good fraction of the available P, around 1/4 in VY CMa (Tenenbaum et al. 2007; Milam et al. 2008) and essentially all available phosphorus in IK Tau (De Beck et al. 2013). 
In IRC +10216 , phosphine is formed somewhere between 1 and $100 R_{*}$ from the star, although it is not clear which is the main formation mechanism. Thermochemical equilibrium calculations indicate that its abundance in the surroundings of the star is very low $\left(<10^{-12}\right.$ relative to $\left.\mathrm{H}_{2}\right)$ and gas phase chemical kinetics models yield no net formation in the outer layers of the envelope (Agúndez et al. 2007). There are various plausible non-equilibrium processes at work in the inner envelope, which may be at the origin of $\mathrm{PH}_{3}$ and other hydrides such as $\mathrm{NH}_{3}$ and $\mathrm{H}_{2} \mathrm{O}$, for which thermochemical equilibrium predicts abundances much lower than observed. Interestingly, the $\mathrm{NH}_{3} / \mathrm{PH}_{3}$ abundance ratio in IRC +10216 ( 200; Hasegawa et al. 2006) is similar to the solar elemental $\mathrm{N} / \mathrm{P}$ abundance ratio, indicating that a similar fraction of $\mathrm{N}$ and $\mathrm{P}$ are locked in $\mathrm{NH}_{3}$ and $\mathrm{PH}_{3}$, respectively. A non-equilibrium chemistry driven by shocks induced by the stellar pulsation has been proposed as a source of water vapor in IRC +10216 (Cherchneff 2011). In this scenario, however, $\mathrm{NH}_{3}$ is formed with a very low abundance and details on whether or not $\mathrm{PH}_{3}$ is formed are not provided. Another mechanism proposed for the formation of hydrides such as $\mathrm{H}_{2} \mathrm{O}$ and $\mathrm{NH}_{3}$ in the inner layers of carbon-rich envelopes such as IRC +10216 is photochemistry driven by the penetration of interstellar UV photons across the clumpy envelope (Decin et al. 2010; Agúndez et al. 2010). In this scenario, $\mathrm{PH}_{3}$ is not efficiently formed, although it is likely due to the lack of relevant chemical kinetics data for phosphorus species. Another possible source of hydrides such as $\mathrm{PH}_{3}$ that is yet to be explored could be through chemical reactions taking place on dust grain surfaces. Which of these mechanisms, if any, is responsible for the formation of $\mathrm{PH}_{3}$ in the ejecta of evolved stars such as IRC +10216 has yet to be investigated.

\section{SUMMARY}

We use the HIFI instrument on board Herschel to observe the $J=2-1$ line of $\mathrm{PH}_{3}$ in IRC +10216 , which, together with the previous observation of the $J=1-0$ line with the IRAM $30 \mathrm{~m}$ telescope, definitively confirms the first identification of $\mathrm{PH}_{3}$ outside the solar system. Excitation and radiative transfer calculations indicate that the observed lines are consistent with phosphine being formed in the circumstellar envelope anywhere between 1 and $100 R_{*}$ from the star with an abundance of $10^{-8}$ relative to $\mathrm{H}_{2}$. The detection of $\mathrm{PH}_{3}$ challenges chemical models, as no obvious formation route has for the moment been found. Prospects to put further constraints on the distribution and origin of $\mathrm{PH}_{3}$ in IRC +10216 with telescope facilities such as ALMA seem challenging. The $J=1-0$ line is blended with a SiS $v=4$ line, which makes it complicated to disentangle the contribution from each line to the spatial distribution of the emission. The $J=2-1$ line at $533.8 \mathrm{GHz}$ cannot be reached from the ground because of the terrestrial atmospheric opacity, and the $J=3-2$ line is also severely blended with a strong line of $\mathrm{SiC}_{2}$. Although $\mathrm{PH}_{3}$ holds a minor fraction of phosphorus in IRC +10216 , it is, together with $\mathrm{HCP}$, one of the major gas phase carriers of phosphorus in the inner circumstellar layers, suggesting that it could also be an important phosphorus species in other astronomical environments. Observations of $\mathrm{PH}_{3}$ in sources other than IRC +10216 , such as CRL 2688, may help us to better understand its formation and the implications for the chemistry of phosphorus in space.
HIFI has been designed and built by a consortium of institutes and university departments from across Europe, Canada, and the United States (NASA) under the leadership of SRON, Netherlands Institute for Space Research, Groningen, The Netherlands, and with major contributions from Germany, France, and the U.S. Consortium members are Canada: CSA, U. Waterloo; France: CESR, LAB, LERMA, IRAM; Germany: KOSMA, MPIfR, MPS; Ireland: NUI Maynooth; Italy: ASI, IFSI-INAF, Osservatorio Astrofísico di Arcetri-INAF; Netherlands: SRON, TUD; Poland: CAMK, CBK; Spain: Observatorio Astronómico Nacional (IGN), Centro de Astrobiología (INTA-CSIC); Sweden: Chalmers University of Technology - MC2, RSS, \& GARD, Onsala Space Observatory, Swedish National Space Board, Stockholm University-Stockholm Observatory; Switzerland: ETH Zurich, FHNW; USA: CalTech, JPL, NHSC. M.A. and J.C. thank Spanish MICINN for funding support through grant CSD2009-00038.

\section{REFERENCES}

Agúndez, M., Biver, N., Santos-Sanz, P., et al. 2014, A\&A, 564, L2 Agúndez, M., Cernicharo, J., \& Guélin, M. 2007, ApJL, 662, L91

Agúndez, M., Cernicharo, J., \& Guélin, M. 2010, ApJL, 724, L133 Agúndez, M., Cernicharo, J., Pardo, J. R., et al. 2008, A\&A, 485, L33 Agúndez, M., Fonfría, J. P., Cernicharo, J., et al. 2012, A\&A, 543, A48 Asplund, M., Grevesse, N., Sauval, A. J., \& Scott, P. 2009, ARA\&A, 47,481

Baldacci, A., Devi, V. M., Rao, K. N., \& Tarrago, G. 1980, JMoSp, 81, 179

Bregman, J. D., Lester, D. F., \& Rank, D. M. 1975, ApJ, 202, 55

Brown, L. R., Sams, R. L., Kleiner, I., et al. 2002, JMoSp, 215, 178

Cazzoli, G., \& Puzzarini, C. 2006, JMoSp, 239, 64

Cernicharo, J. 2012, in European Conference on Laboratory Astrophysics, EAS Publication Series, Vol. 58, ed. C. Stehlé, C. Joblin, \& L. d'Hendecourt (Cambridge: Cambridge Univ. Press), 251

Cernicharo, J., Daniel, F., Castro-Carrizo, A., et al. 2013, ApJL, 778, L25

Cernicharo, J., Guélin, M., \& Kahane, C. 2000, A\&AS, 142, 181

Cernicharo, J., Waters, L. B. F. M., Decin, L., et al. 2010, A\&A, 521, L8

Cernicharo, J., Yamamura, I., González-Alfonso, E., et al. 1999, ApJL, 526, L41

Cherchneff, I. 2011, A\&A, 526, L11

Crovisier, J., Bockelée-Morvan, D., Colom, P., et al. 2004, A\&A, 418, 1141

Danby, G., Flower, D. R., Valiron, P., et al. 1988, MNRAS, 235, 229

Davies, P. B., Neumann, R. M., Wofsy, S. C., \& Klemperer, W. 1971, JChPh, 55,3564

De Beck, E., Kamiński, T., Patel, N. A., et al. 2013, A\&A, 558, A132

Decin, L., Agúndez, M., Barlow, M. J., et al. 2010, Natur, 467, 64

Fusina, L., \& Di Lonardo, G. 2000, JMoSt, 517, 67

Guélin, M., Cernicharo, J., Paubert, G., \& Turner, B. E. 1990, A\&A, 230, L9

Halfen, D. T., Clouthier, D. J., \& Ziurys, L. M. 2008, ApJL, 677, L101

Hasegawa, T. I., Kwok, S., Koning, N., et al. 2006, ApJ, 637, 791

Keady, J. J., \& Ridgway, S. T. 1993, ApJ, 406, 199

Larson, H. P., Fink, U., \& Treffers, R. R. 1977, ApJ, 211, 972

Machin, L., \& Roueff, E. 2005, J. Phys. B: Mol. Opt. Phys., 38, 1519

Maciá, E. 2005, Chem. Soc. Rev., 34, 691

MacKay, D. D. S., \& Charnley, S. B. 2001, MNRAS, 325, 545

Milam, S. N., Halfen, D. T., Tenenbaum, E. D., et al. 2008, ApJ, 684, 618

Monnier, J. D., Danchi, W. C., Hale, D. S., et al. 2000, ApJ, 543, 868

Moreno, R., Marten, A., \& Lellouch, E. 2009, DPS, 41, 28.02

Müller, H. S. P. 2013, JQSRT, 130, 335

Müller, H. S. P., Cernicharo, J., \& Agúndez, M. 2012, JMoSp, 271, 50

Sousa-Silva, C., Yurchenko, S. N., \& Tennyson, J. 2013, JMoSp, 288, 28

Tarrago, G., Ulenikov, O. N., \& Poussigue, G. 1984, JPhys, 45, 1429

Tenenbaum, E. D., Woolf, N. J., \& Ziurys, L. M. 2007, ApJL, 666, L29

Tenenbaum, E. D., \& Ziurys, L. M. 2008, ApJL, 680, L121

Turner, B. E., \& Bally, J. 1987, ApJL, 321, L75

Turner, B. E., Tsuji, T., Bally, J., Guélin, M., \& Cernicharo, J. 1990, ApJ, 365,569

Weisstein, E. W., \& Serabyn, E. 1996, Icar, 123, 23

Yurchenko, S. N., Carvajal, M., Thiel, W., \& Jensen, P. 2006, JMoSp, 239, 71

Ziurys, L. M. 1987, ApJL, 321, L81 